\title{
Minireview
}

\section{Adaptation beyond the Stress Response: Cell Structure Dynamics and Population Heterogeneity in Staphylococcus aureus}

\author{
KAZUYA MORIKAWA ${ }^{*}$, RYOSUKE L. OHNIWA ${ }^{1}$, TOSHIKO OHTA ${ }^{1 \dagger}$, YOSHIKAZU TANAKA ${ }^{2}$, \\ KUNIO TAKEYASU ${ }^{3}$, and TAREK MSADEK ${ }^{4}$ \\ ${ }^{1}$ Graduate School of Comprehensive Human Sciences, University of Tsukuba, Tsukuba 305-8575, Japan; ${ }^{2}$ Faculty of Advanced \\ Life Science, Hokkaido University, Sapporo 060-0810, Japan; ${ }^{3}$ Graduate School of Biostudies, Kyoto University, Kyoto 606-8502, \\ Japan; and ${ }^{4}$ Biology of Gram-Positive Pathogens, Institut Pasteur, CNRS URA 2172, 75724 Paris Cedex 15, France
}

(Received March 3, 2010—Accepted April 4, 2010—Published online April 19, 2010)

Staphylococcus aureus, a major opportunistic pathogen responsible for a broad spectrum of infections, naturally inhabits the human nasal cavity in about $30 \%$ of the population. The unique adaptive potential displayed by $S$. aureus has made it one of the major causes of nosocomial infections today, emphasized by the rapid emergence of multiple antibiotic-resistant strains over the past few decades. The uncanny ability to adapt to harsh environments is essential for staphylococcal persistence in infections or as a commensal, and a growing body of evidence has revealed critical roles in this process for cellular structural dynamics, and population heterogeneity. These two exciting areas of research are now being explored to identify new molecular mechanisms governing these adaptational strategies.

Key words: Staphylococcus aureus, nucleoid, membrane, variant, population heterogeneity

\section{Introduction}

Staphylococcus belongs to the Gram-positive Firmicutes Bacillales group that also includes Bacillus, and Listeria spp. $S$. aureus is a commensal bacterium that naturally inhabits the nasal cavity of mammals, but it is also an opportunistic pathogen responsible for a broad spectrum of infections ranging from food poisoning and superficial skin abscesses to more serious diseases such as pneumonia, meningitis, osteomyelitis, septicemia and toxic shock syndrome. It has acquired resistance to a wide variety of antibiotics (39), and methicillin-resistant strains (MRSA), the most common cause of nosocomial infections, are now spreading into the community (12).

$S$. aureus has evolved multiple characteristics allowing it to colonize the outer surfaces of the host, including the anterior nares. In the nasal cavity, lysozyme, the first barrier in our defense systems, is secreted to destroy invading bacteria, but staphylococci have acquired resistance to lysozyme by modifying their peptidoglycan structure through $O$-acetylation $(6$, $35)$. Drastic changes in osmotic pressure are also a major threat to bacterial survival on the outer surface, though the ability of staphylococci to grow over a broad range of salt concentrations is well documented. These characteristics combine to allow staphylococci to predominate on the host's outer surfaces.

This highly successful pathogen is also equipped with a variety of tools for survival during infection, such as factors to colonize host tissues (18), to evade the innate immune system (27), to acquire iron (49), or to form biofilms (69).

\footnotetext{
* Corresponding author. E-mail: morikawa@sakura.cc.tsukuba.ac.jp; Tel \& Fax: +81-29-853-3928.

† Present address: Japan Aerospace Exploration Agency, Tsukuba Space Center, Tsukuba 305-8505, Japan
}

Categorized as virulence factors and extensively reviewed elsewhere, they likely play a part in facilitating transmission and sustaining a commensal relationship under proper regulatory conditions, which $S$. aureus might have evolved to prevent causing an infection in a healthy host (10).

One prominent feature of $S$. aureus adaptation to stress is its ability to form 'variants' both in vivo and in vitro. Studies on some biochemical characteristics of variants have explained the mechanisms of their successful adaptation (see below), but the specific mechanisms involved in generating such population heterogeneity or variants remains poorly understood for the most part.

In this review, we start with 1) an overview of the cellular stress responses, and then focus on two recently emphasized concepts, namely 2) the dynamics of cell structure/components, and 3) population heterogeneity or the formation of variants. The interplay in multiple systems including the well established cellular responses are also discussed, in a search for clues to expand our understanding of the uncanny adaptational capabilities of $S$. aureus.

\section{Classical stress response, at a glance}

The ability to cope with stress has been studied as the cellular response to stress and the resultant expression of stress-responsive factors. Many studies helped establish the general concept that signal transduction and the regulation of gene expression play key roles in stress response/adaptation (19).

Sigma factors. As in most bacteria, sigma factors are master regulators of transcription. Sigma factors are a subunit of RNA polymerase involved in the recognition of specific promoter sequences to initiate site-specific transcription $(55,90)$. Generally, bacteria have multiple sigma factors each of which regulates a series of genes often constituting 
the cognate biological system (33). So far, four sigma factors have been identified in $S$. aureus. SigA is the primary sigma factor. It is conserved in all bacteria and responsible for the expression of house-keeping genes (23). SigB is the alternative sigma factor for general responses to stress (24). Its levels are constant throughout the growth phase (38), but SigB can be activated under unusual $\mathrm{pH}$ conditions, heat shock (transient), high osmolarity, and later growth phase through the 'partner switching' mechanism, although the precise mechanism for signal transduction to the SigB regulators in $S$. aureus is not known (86). Two additional sigma factors, SigH (56) and SigS (79), were identified later, but they are only weakly expressed under ordinary growth conditions, and their physiological roles remain elusive.

Two-component systems (TCSs). Two-component systems (TCSs) elegantly combine sensing, transducing and transcriptional activation modules within two proteins. The common phosphotransfer mechanism of signal transduction is reflected by the high degree of conservation within the transducing modules, whereas the sensing and response modules display the variability required for signal/response specificity. The first component, a histidine protein kinase, is autophosphorylated at a conserved histidine residue in an ATP-dependent reaction. In a second step, the phosphoryl group is transferred to an aspartate residue in the conserved amino-terminal domain of the second component, the response regulator. Regulatory pathways involving TCSs are often highly interconnected and form complex signal transduction networks (59). Most $S$. aureus strains are endowed with sixteen sets of genes encoding two-component systems, with an additional one present in the staphylococcal cassette chromosome mec of MRSA, linked to induction of methicillin resistance. This sophisticated arsenal of environmental monitoring proteins could, in part, explain the highly adaptive nature of $S$. aureus. Many of these systems remain to be characterized, although several have been shown to play a role in virulence (AgrC/AgrA, SaeS/SaeR) (8), resistance to antibiotics (VraS/VraR, GraS/GraR) $(35,43)$, or adaptations to environmental changes such as oxygen pressure (SrrA/SrrB) and as targets of drug development $(26,30)$. The best-studied $S$. aureus TCS is agrAC that constitutes a peptide quorum-sensing system, a key regulatory system for virulence gene expression.

SarA. SarA (staphylococcal accessory regulator) and its homologues are extensively studied regulators (16). They share a winged helix DNA-binding domain and participate in the regulation of many genes. SarA prefers to bind AT-rich DNA, but lacks a clear consensus target sequence. It was recently found that SarA is present at $\sim 50,000$ copies per CFU (29), and was suggested that SarA is the functional counterpart of Escherichia coli global regulators such as $\mathrm{Hu}$ and IHF, rather than a classical transcription factor.

Specific regulatory systems. Classical transcription factors constitute many of the unique staphylococcal regulatory systems. The heat shock-evoked response of chaperone gene expression controlled by $\mathrm{CtsR}$ and HrcA is among the bestdocumented systems regarding their molecular mechanisms and evolutionary aspects $(13,25,42,68)$. In Bacillus subtilis, heat shock genes are classified into four groups according to the regulators responsible for their expression: Class I is controlled by the HrcA repressor, Class II by SigB, Class III by CtsR, and Class IV by others. The HrcA repressor recognizes the CIRCE operator sequence. CtsR recognizes a tandem heptanucleotide direct repeat. Class I genes including classical chaperone genes (dnaK, groES, groEL) and Class III genes including $c l p$ genes are distinct in B. subtilis. However, in $S$. aureus, the entire HrcA regulon is embedded within the CtsR regulon, with the synthesis of HrcA itself controlled by CtsR (13). Indeed, the CtsR protein of $S$. aureus binds specifically to the $\operatorname{clp} P, \operatorname{clp} C, \operatorname{clp} B, \operatorname{dnaK}$ and groESL regulatory regions, and both $\mathrm{CtsR}$ and $\operatorname{HrcA}$ bind to the $S$. aureus dnaK and groESL promoter regions (13, 28). Other examples, described below, involve evolutionary distinct transcription factors to regulate the same gene or phenomenon: PerR/OxyR-based regulation of $d p s$ family genes, and ComK/SigH-based regulation of com genes. Thus, bacteria have evolved species-specific regulatory systems that differ even among close relatives such as $S$. aureus and B. subtilis.

Through such master/global regulators or transcription factors, the gene expression profile is though to be modulated to adapt to given environments. For example, oxidative stress regulons include enzymes that detoxify or reduce the level of oxidative stress (20), and the agr quorum-sensing system induces the expression of exo-enzymes that degrade host tissues to utilize them as nutrients (15). In addition to the direct function of virulence factors, additional layers of adaptation include the dynamic change of cell architectural components and the resultant conversion of the cell's physical characteristics, which are the focus of the next chapter.

\section{Cell architecture dynamics: Cell membrane components}

Although staphylococci are immobile and cannot form spores, the cell architecture including the nucleoid and cell membrane undergo dynamic changes when responding and adapting to environmental stress.

Cardiolipin. A general strategy among bacteria is to change phospholipid composition in response to growth phase or environmental stress such as osmolality (74), $\mathrm{pH}$ $(31,51)$, temperature and the presence of organic solvents $(7,73)$. In the 1970 's, the molecular mechanism of staphylococcal salt resistance was studied, focusing on a phospholipid, cardiolipin (CL), which constitutes about $30 \%$ of the cell membrane in the stationary phase of growth (80). Two phosphatidylglycerols are enzymatically connected through the glycerol backbone to form CL, which carries four fatty acid tails and two negative charges (77). CL can stabilize a membrane against changes of osmotic stress when included in liposomes (60). Recently, CL was demonstrated to be important for $E$. coli and B. subtilis to grow at high salinity $(47,72)$. In contrast, $S$. aureus does not require CL to grow in high salinity medium (Tsai et al., submitted). Instead, CL is important for long-term survival under high salinity or sudden hypertonic shock. Two genes were found to encode functional CL synthases. The physiological significance of the differential usage of the two homologous enzymes is still unclear, but we recently found that one of them functions specifically under conditions of stress such as high salinity, high temperature, and high $\mathrm{pH}$. Further detailed analysis 
focusing on the distinct roles of the two CL synthases is required to understand the role(s) of CL dynamics in $S$. aureus.

Carotenoid and others. The cell membrane also contains a carotenoid pigment, staphyloxanthin, conferring on $S$. aureus its characteristic yellow or orange color. $S$. aureus was named after this characteristic ('aureus' stands for golden in Latin), although recent clinical isolates often lack the pigment. The genes for staphyloxanthin biosynthesis $(\operatorname{crtM}$, and $\operatorname{crtN})(87)$ are under the control of SigB and the yellow color develops through prolonged growth under aerobic conditions. Liu et al. indicated that staphyloxanthin contributes to resistance to oxidative stress produced by host phagocytes (46).

Changes in other cell membrane/cell wall components (e.g., lysyl-phosphatidylglycerol, teichoic acid, and cell wall thickness) are also important for adaptation to a variety of agents such as cationic antimicrobial peptides (70) and the clinically important antibiotic vancomycin (21).

\section{Cell architecture dynamics: Nucleoid}

Bacterial genomic DNA with a size of $\sim 1 \mathrm{~mm}$ is packed in a cell of $\sim 1 \mu \mathrm{m}$ diameter together with nucleoid-associated proteins (NAPs), RNA, and others $(66,67)$. This complex, termed the nucleoid, is the site of a series of events important for maintaining genome functions, such as duplication, segregation, gene expression, repair, and insertion/excision of mobile genetic elements. There exist several hundred NAPs and our recent proteomic analysis found that the majority detected in the nucleoid fraction vary among genera (Ohniwa et al., in preparation). Furthermore, they are highly variable depending on growth phase: roughly $70 \%$ are replaced from the $\log$ to stationary phase in $S$. aureus. The nucleoid from lysed cells is usually a fibrous structure varying in thickness when observed by the 'on-substrate lysis' method with atomic force microscopy regardless of the growth phase (84), but the physical characteristics of the nucleoid change in response to oxidative stress, where the MrgA protein plays a key role $(57,58)$.

Species-specific expression of Dps/MrgA. MrgA, endoced by $\operatorname{mrg} A$ (metal ion responsive gene A) (14), is a homologue of $E$. coli Dps. Dps is a member of the Febinding protein family that forms dodecameric complexes (3, 32). Dps can bind DNA and protect it against oxidative stress (50), nuclease cleavage, UV damage, thermal shock (62) and acidic environments (17). Dps is one of very few nucleoid proteins that are conserved in many bacterial species (84), including $S$. aureus. However, the manner in which Dps is induced to express depends on the bacterial species. Fig. 1 summarizes the difference in Dps expression between $S$. aureus and E. coli. The E. coli Dps is the most abundant protein in the tightly condensed nucleoid at the stationary phase $(41,89)$, whereas the staphylococcal MrgA is not expressed towards the stationary phase and the nucleoid sustains the fibrous structures (57). The nucleoid of $S$. aureus undergoes clogging following molecular genetic manipulation ( $m r g A$ overexpression or perR knockout) but it allows cell proliferation, indicating that the MrgA-dependent nucleoid clogging can permit gene expression and genome
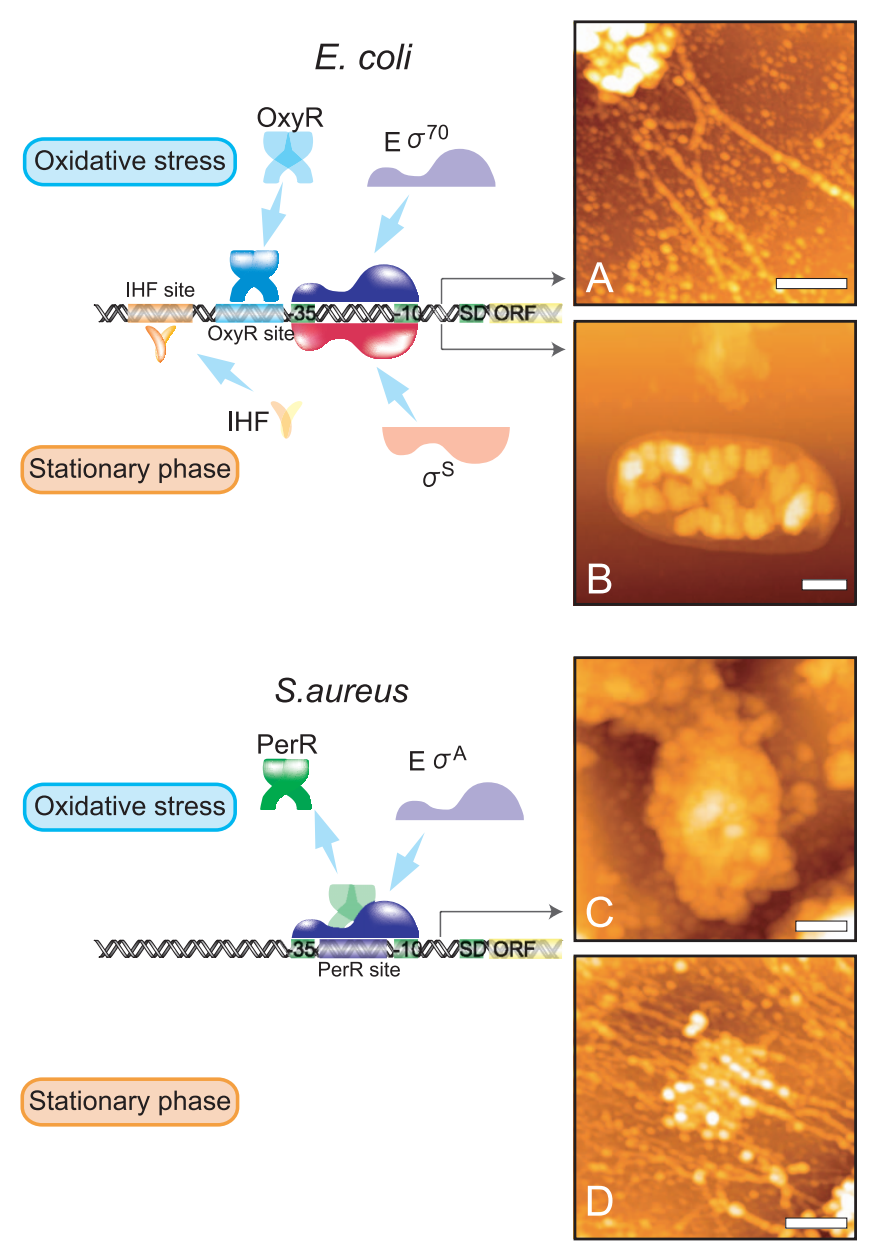

Fig. 1. Regulatory mechanisms of $d p s / m r g A$ and the dynamics of nucleoid structure in $E$. coli and $S$. aureus. In $E$. coli, $d p s$ gene expression is induced towards the stationary phase by IHF, which binds to the -50 region together with the $\sigma^{\mathrm{s}}$-containing holoenzyme $\left(E \sigma^{\mathrm{s}}\right)$. OxyR stimulates $d p s$ gene expression under oxidative stress. In $S$. aureus, only the PerR pathway exists due to the lack of the stationary phase pathway. OxyR and PerR are evolutionary distinct, and OxyR acts as an activator, while PerR is a repressor whose release under oxidative stress induces $m r g A$ expression. The fibrous nucleoid in the $\log$ phase undergoes tight compacting towards the stationary phase in E. coli (panel B), but not under oxidative stress (panel A) due to the presence of the E. coli-specific Fis that interferes with Dps-dependent compaction. The nucleiod of $S$. aureus is clogged under oxidative stress through induction of MrgA protein synthesis (panel C), but not in the stationary phase (panel D). Scale bar $=500 \mathrm{~nm}$.

duplication/segregation (57). Both E. coli dps and S. aureus $m r g A$ are induced to express by oxidative stresses via evolutionary distinct transcription factors, both of which can sense oxidative stress directly. The oxidative stress then causes nucleoid clogging through the induction of MrgA in $S$. aureus but not in $E$. coli, due to the presence of $E$. colispecific Fis proteins that inhibit the Dps dependent genome condensation (65).

MrgA-dependent oxidative stress resistance: Fe binding or DNA binding? The $S$. aureus nucleoid clogged by MrgA is biologically active in contrast to the E. coli nucleoid compacted by Dps in the stationary phase, or more strikingly, the genome protected by acid soluble proteins in spores of B. subtilis $(54,78)$. Consequently there is a need to evaluate whether staphylococcal nucleoid clogging has DNA protec- 
tive roles similar to the physical protection provided by $E$. coli Dps (or acid soluble proteins in spores). The fact that $m r g A$ is essential to cope with oxidative stress suggests that one or both of its Fe-binding and DNA-binding activity could contribute to oxidative stress resistance. Scavenging free iron is important to reduce the Fenton reaction that generates the hydroxyl radical from ferrous iron $\left(\mathrm{Fe}^{2+}\right)$ and hydrogen peroxide (37). In Streptococcus mutans, where the Dps-family Dpr protein can bind iron but not DNA, Febinding activity was demonstrated to be critical to cope with oxidative stress (91). However, no Dps family members exhibit DNA-binding activity without Fe-binding activity, leaving open to question the relevance of physical nucleoid clogging and oxidative stress resistance. Currently, we assume that Fe-binding by MrgA is the primary requirement to cope with oxidative stress, and its DNA-binding activity would keep it close to the genome, thereby reducing the Fe concentration around the DNA. This assumption is based on the fact that nucleic acids bind iron well, and the hydroxyl radical is unlikely to spread far from its origin in cellular environments (37). Our recent preliminary results indicated that a mutated MrgA derivative that can bind DNA but cannot bind Fe was unable to protect $S$. aureus cells against oxidative stress (unpublished result). Although it is currently difficult to demonstrate our assumption, a detailed structural analysis of the complex of MrgA-Fe-DNA might provide a clue.

\section{Population heterogeneity}

Phenotypic heterogeneity. Diverse subpopulations confer to cells a better chance of surviving a variety of conditions or stress than a homogeneous population. A heterogeneous subpopulation can be generated by diversified gene expression through changes in a variety of cellular factors such as signal transducers, transcription factors, nucleoid proteins including global regulators, and non-protein-coding RNAs (1). The existence of heterogeneous cell populations is well documented regarding antibiotic resistance in $S$. aureus, and a 'population analysis' is often used to evaluate the pattern of the successive distribution of resistance in a genetically identical cell population (36). On the other hand, the cell population from a single clone can also exhibit completely distinct phenotypes. In $B$. subtilis, for example, the competence-related genes are expressed in a stochastic manner. The molecular basis for this stochastic expression is the regulatory network controlling the ComK transcription factor, where the positive and negative feedback loops are interconnected $(48,82)$. Although environmental signals are integrated into this regulatory network, some 'noise' can shift the mode of this network to switch on ComK activity in a stochastic manner. The competence gene homologues in $S$. aureus also undergo stochastic expression, although these genes are regulated by the secondary sigma factor SigH, which is evolutionary distinct from ComK (Morikawa et al., in preparation).

Genetic variation. In contrast to the phenotypic heterogeneity in genetically homogeneous cell populations, heterogeneity in bacterial populations is often generated through genetic changes. The genetic change can be spontaneous or
Table 1. Examples of population heterogeneity in S. aureus

\begin{tabular}{|c|c|c|}
\hline Types & Notes & References \\
\hline Antibiotic resistance & & $(36)$ \\
\hline \multicolumn{3}{|l|}{ Variant } \\
\hline $\begin{array}{l}\text { small colony variants } \\
\text { (SCVs) }\end{array}$ & $\begin{array}{l}\text { deficient in electron } \\
\text { transport }\end{array}$ & $(5,71)$ \\
\hline L-form & $\begin{array}{l}\text { w/o cell wall, stable or } \\
\text { unstable }\end{array}$ & (2) \\
\hline others & $\begin{array}{l}\text { variants resistant to e.g. } \\
\text { antibiotics }\end{array}$ & (11) \\
\hline \multicolumn{3}{|l|}{ Phase variation* } \\
\hline hyperhemolytic phenotype & & $(92)$ \\
\hline length of MapW protein & $\begin{array}{l}\text { poly }(\mathrm{A}) \text { tract in coding } \\
\text { sequence }\end{array}$ & (9) \\
\hline biofilm formation & $\begin{array}{l}\text { IS256 insertion in } i c a \text { or } \\
\quad \text { sarA }\end{array}$ & $(40,85)$ \\
\hline protease activity & & $(85)$ \\
\hline \multicolumn{3}{|l|}{ Other } \\
\hline SJ duplication in $\operatorname{sig} H$ & see Fig. 2 & unpublished \\
\hline
\end{tabular}

* a comprehensive list of phase variation in bacteria is available in reference (88).

inducible, which is sometimes difficult to distinguish. $S$. aureus has multiple methods of generating heterogeneity as described below (Table 1). One method is the formation of variants under stress, and among the best documented variants are small colony variants $(\mathrm{SCVs})(5,71)$ and L-form variants.

Small colony variants (SCVs). Small colony variants (SCVs) can be recovered from patients with cystic fibrosis and chronic/persistent infections, and are thought to be an adaptive form to sustain an infection in the host. SCVs are able to persist within non-professional phagocytes, being protected against host bactericidal factors. It is also possible to select or generate SCVs in vitro by using aminoglycoside and the Pseudomonas aeruginosa exoproduct 4-hydroxy-2heptylquinoline- $N$-oxide (HQNO) (53): $P$. aeruginosa is also often isolated from cystic fibrosis patients. In many cases, SCVs are deficient in the electron transport system, such as the pathways for producing hemin, menadione and thiamine, and thereby exhibit slow growth. Indeed, the disruption of genes involved in hemin or menadione biosynthesis induces the SCV phenotype. Here it should be noted that aminoglycoside is transported into cells via a proton gradient generated by the electron transport system (83), and HQNO specifically blocks electron transport in Gram-positive bacteria (44). The mutator phenotype increases the frequency with which SCVs form. In addition, it is likely that the formation of SCVs is regulated by environmental signals. Mitchell et al. have reported that the general stress response sigma factor, SigB, plays important role(s) in the formation of SCVs induced by aminoglycoside (52) and by HQNO (53), suggesting interplay between the stress response system and the variant forming process.

L-form. The L-form is another special variant generated by $S$. aureus as well as by other bacteria (2). It lacks cell walls, and requires proper osmotic pressure to be isolated from patients. L-forms can also be generated in vitro by long-term (several days) incubation with cell wall-perturbing agents such as $\beta$-lactams and lysostaphin. The membrane of L-form cells is thought to be equipped with certain features 
that can support cell growth and survival without rigid cell wall. A previous study found that a certain L-form strain increased CL content (34). Our recent molecular genetic study found that one of the two CL synthase genes (SA1891) is important for generating the L-form, but we found that a cls $1 /$ cls 2 double mutant lacking CL can still generate L-form cells albeit with reduced frequency (Tsai et al., submitted). This suggests the existence of CL-independent mechanisms. It is reasonable to think that multiple mechanisms cooperate to generate the L-form variant. It is worth noting that biochemical characteristics such as catalase, coagulase, lipase, fibrinolysin, haemolysin, and enterotoxin production can change during L-form generation and reversion (22, 75, 76, 81 ), but none of these is a common phenotype for L-form variants, suggesting that the L-form is generated concomitant with drastic phenotypic conversions, where the increase in CL content may be an effective, but dispensable, way to stabilize the membrane. It should also be noted that the membrane of L-forms contains cholesterol $(34,63)$. Since the $S$. aureus genome lacks any apparent genes for cholesterol synthesis, it has not been concluded whether $S$. aureus can develop a sterol producing capability. It is interesting that certain inhibitors for human cholesterol synthase can prevent the function of CrtM responsible for carotenoid synthesis (45). It is not known whether the crtM gene or CrtM enzyme is altered in cholesterol-producing L-form variants.

Phase variation. S. aureus also exhibits population heterogeneity through 'phase variation'. Phase variation is a phenomenon whereby the expression of a certain phenotype is converted at frequencies higher than $10^{-5}$ per cell per generation, usually in a reversible manner, and is thought to be a major adaptative strategy in bacteria $(4,88)$. Phase variants are generated by a variety of interesting genetic (mutation, site-specific recombination, homologous recombination, simple sequence DNA repeat tracts) or epigenetic changes [reviewed in (4)]. Reported or postulated phase variations in $S$. aureus include a hyperhemolytic phenotype produced by an unidentified mechanism (92), variable lengths of the MapW protein (major histocompatibility complex MHC class II analogue) (9), and the on/off switch for the formation of a biofilm (40).

The Map-like protein genes are categorized into two groups, one of which, including the $S A 1751$ gene in strain $\mathrm{N} 315$, has a poly(A) tract within the coding sequence. The length of the poly(A) tract in SA1751 is 10 bases, introducing a stop codon immediately after the tract. On the other hand, other strains have variable lengths of the poly(A) tract, and 8 or 11 adenines result in the full-length translation of the protein. The shift in the poly(A) tract's length is considered to be brought about by the slipped-strand mispairing mechanism.

Phase variation in the biofilm switch is mediated by the reversible insertion/excision of the insertion sequence, IS256, in biofilm essential genes such as the ica locus (40) and $\operatorname{sar} A$ (85). The insertion of IS256 was suggested not to be random in the genome, because the formation of variants is observed only for the biofilm and protease phenotypes but not for others such as coagulase, hemolysin, and lipase (85). It is interesting that SigB regulates the frequency of IS256 transposition (85), though the precise mechanism is not known. In general, transposition is regulated/affected by a variety of host factors that differ depending on the mobile element (61).

Population heterogeneity by SigH. Recently, a new system generating population heterogeneity was found involving SigH activation (Fig. 2) (Morikawa et al., in preparation). As mentioned above, SigH activity is undetectable when the population is analyzed as a whole, e.g., by examining SigH protein levels by Western blotting (56). This is because SigH production is restricted to a minor cell population. SigH is activated by two distinct mechanisms: one involves posttranscriptional regulation, and the other is transient genetic

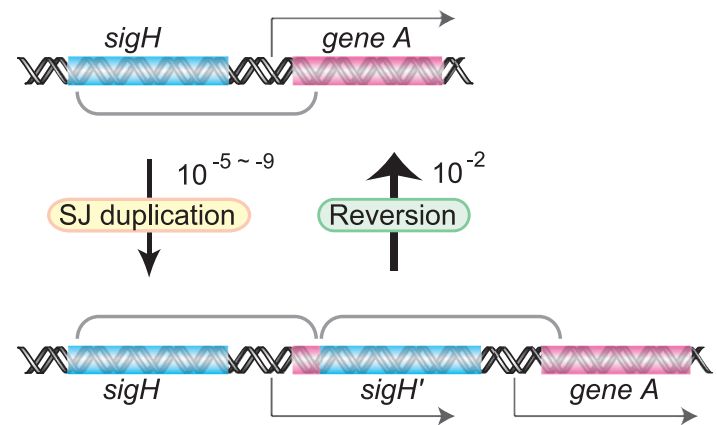

Fig. 2. The activation of $\operatorname{sigH}$ by Short Junction (SJ) duplication. SJduplication occurs at the sigH locus and the region encompassed by short direct repeats is duplicated at low frequencies $\left(10^{-5}-10^{-9}\right)$. The resultant new chimeric sigH gene, that is the in-frame fusion of the downstream gene and sigH, is expressed and the SigH protein is produced so as to induce its regulon. The duplication ceases at a frequency of about $10^{-2}$. SigH can be also produced through post-transcriptional regulation without the SJ duplication system, but in both cases, SigH activation remains restricted to a minor cell population.

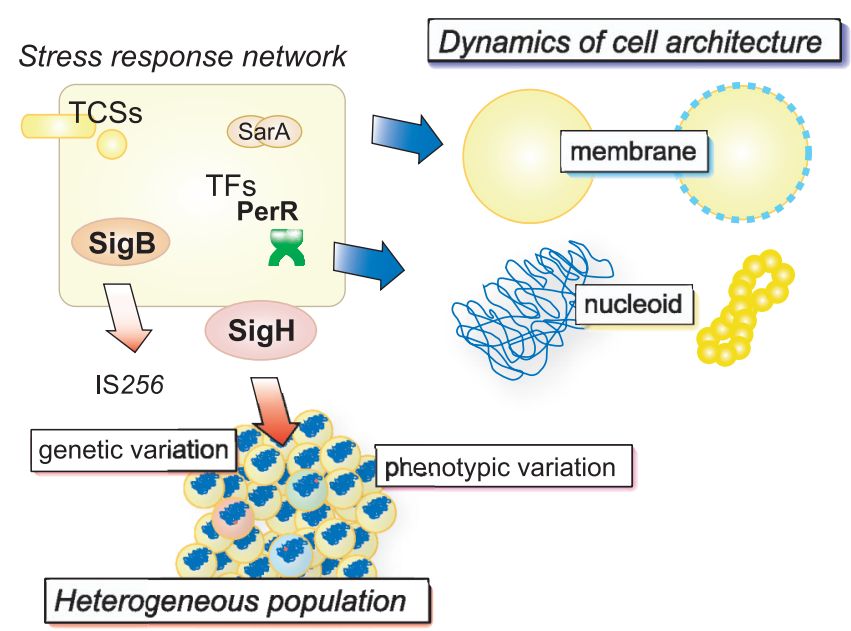

Fig. 3. Schematic representation of the adaptational strategies detailed in this review. Stress signals are sensed and transduced through general or species-specific regulatory networks so as to modulate gene expression. A part of the stress regulon plays an important role in cell architectural dynamics. On the other hand, generating a heterogeneous cell population is beneficial according to the 'insurance hypothesis'. Heterogeneity is generated either by uneven/stochastic gene expressions or by genetic conversions. Alternative sigma factors, which are generally responsible for stress responses, can affect population heterogeneity. Variants of cell architecture include SCVs, L-form, biofilm variants, antibiotic-resistant variants, etc. 
change. In the latter, short-junction (SJ) duplication occurs at the $\operatorname{sig} H$ locus so as to generate a new in-frame fusion gene at frequencies of $10^{-5}-10^{-9}$ in a spontaneous manner. The resultant $\operatorname{sig} H$ fusion gene is expressed under the control of promoter and translation initiation signals originating from the partner gene. However, this state is genetically unstable, and the duplication is cured at a frequency of $\sim 10^{-2}$, probably helping to maintain the original state (without the duplication) in the majority of the population. This is similar to phase variation, but the frequency of the duplication is lower. It will be of great interest to examine whether the SJ duplication can randomly occur in the genome at direct repeat sites, and if so, to examine how much this mechanism contributes to the adaptive ability of $S$. aureus or other bacteria, which will be the focus of our future work.

\section{Conclusions}

In this review, two recently developed aspects of stress adaptation were highlighted. The findings on 'cell architecture dynamics' reveal the importance of structural/physical features in understanding stress adaptation. The 'population heterogeneity' indicates the necessity for further analysis of their still obscure molecular mechanisms. We emphasize that there is interdependency or crosstalk among the distinct systems: e.g., relevance of the CL content and L-form variant formation, SigB regulated formation of IS256 dependent variants, and the direct generation of population heterogeneity by SigH (Fig. 3). Such multi-layered interconnected survival strategies, together with the general or specialized horizontal gene transfer systems $(7,64)$, are key features in understanding how $S$. aureus has developed as such a successful pathogen.

\section{References}

1. Abu-Qatouseh, L.F., S.V. Chinni, J. Seggewiss, R.A. Proctor, J. Brosius, T.S. Rozhdestvensky, G. Peters, C. von Eiff, and K. Becker. 12 February 2010. Identification of differentially expressed small non-protein-coding RNAs in Staphylococcus aureus displaying both the normal and the small-colony variant phenotype. J. Mol. Med. doi:10.1007/s00109-010-0597-2.

2. Allan, E.J., C. Hoischen, and J. Gumpert. 2009. Bacterial L-forms. Adv. Appl. Microbiol. 68:1-39.

3. Almiron, M., A.J. Link, D. Furlong, and R. Kolter. 1992. A novel DNA-binding protein with regulatory and protective roles in starved Escherichia coli. Genes. Dev. 6:2646-2654.

4. Bayliss, C.D. 2009. Determinants of phase variation rate and the fitness implications of differing rates for bacterial pathogens and commensals. FEMS Microbiol. Rev. 33:504-520.

5. Becker, K., G. Bierbaum, C. von Eiff, et al. 2007. Understanding the physiology and adaptation of staphylococci: a post-genomic approach. Int. J. Med. Microbiol. 297:483-501.

6. Bera, A., S. Herbert, A. Jakob, W. Vollmer, and F. Gotz. 2005. Why are pathogenic staphylococci so lysozyme resistant? The peptidoglycan O-acetyltransferase OatA is the major determinant for lysozyme resistance of Staphylococcus aureus. Mol. Microbiol. 55:778-787.

7. Bernal, P., A. Segura, and J.-L. Ramos. 2007. Compensatory role of the cis-trans-isomerase and cardiolipin synthase in the membrane fluidity of Pseudomonas putida DOT-T1E. Environ. Microbiol. 9:1658-1664.

8. Bronner, S., H. Monteil, and G. Prëvost. 2004. Regulation of virulence determinants in Staphylococcus aureus: complexity and applications. FEMS Microbiol. Rev. 28:183-200.

9. Buckling, A., J. Neilson, J. Lindsay, R. ffrench-Constant, M. Enright,
N. Day, and R.C. Massey. 2005. Clonal distribution and phasevariable expression of a major histocompatibility complex analogue protein in Staphylococcus aureus. J. Bacteriol. 187:2917-2919.

10. Camargo, I.L., and M.S. Gilmore. 2008. Staphylococcus aureusprobing for host weakness? J. Bacteriol. 190:2253-2256.

11. Campion, J.J., P. Chung, P.J. McNamara, W.B. Titlow, and M.E. Evans. 2005. Pharmacodynamic modeling of the evolution of levofloxacin resistance in Staphylococcus aureus. Antimicrob. Agents Chemother. 49:2189-2199.

12. Chambers, H.F., and F.R. Deleo. 2009. Waves of resistance: Staphylococcus aureus in the antibiotic era. Nat. Rev. Microbiol. 7:629-641.

13. Chastanet, A., J. Fert, and T. Msadek. 2003. Comparative genomics reveal novel heat shock regulatory mechanisms in Staphylococcus aureus and other Gram-positive bacteria. Mol. Microbiol. 47:10611073.

14. Chen, L., and J.D. Helmann. 1995. Bacillus subtilis MrgA is a Dps(PexB) homologue: evidence for metalloregulation of an oxidative-stress gene. Mol. Microbiol. 18:295-300.

15. Cheung, A.L., A.S. Bayer, G. Zhang, H. Gresham, and Y.-Q. Xiong. 2004. Regulation of virulence determinants in vitro and in vivo in Staphylococcus aureus. FEMS Immunol. Med. Microbiol. 40:1-9.

16. Cheung, A.L., K.A. Nishina, M.P. Trotonda, and S. Tamber. 2008. The SarA protein family of Staphylococcus aureus. Int. J. Biochem. Cell Biol. 40:355-361.

17. Choi, S.H., D.J. Baumler, and C.W. Kaspar. 2000. Contribution of $d p s$ to acid stress tolerance and oxidative stress tolerance in Escherichia coli O157:H7. Appl. Environ. Microbiol. 66:3911-3916.

18. Clarke, S.R., and S.J. Foster. 2006. Surface adhesins of Staphylococcus aureus. Adv. Microb. Physiol. 51:187-224.

19. Clements, M.O., and S.J. Foster. 1999. Stress resistance in Staphylococcus aureus. Trends Microbiol. 7:458-462.

20. Cosgrove, K., G. Coutts, I.M. Jonsson, A. Tarkowski, J.F. Kokai-Kun, J.J. Mond, and S.J. Foster. 2007. Catalase (KatA) and alkyl hydroperoxide reductase $(\mathrm{AhpC})$ have compensatory roles in peroxide stress resistance and are required for survival, persistence, and nasal colonization in Staphylococcus aureus. J. Bacteriol. 189:1025-1035.

21. Cui, L., A. Iwamoto, J.-Q. Lian, H.-M. Neoh, T. Maruyama, Y. Horikawa, and K. Hiramatsu. 2006. Novel mechanism of antibiotic resistance originating in vancomycin-intermediate Staphylococcus aureus. Antimicrob. Agents Chemother. 50:428-438.

22. Czop, J.K., and M.S. Bergdoll. 1970. Synthesis of enterotoxin by L-forms of Staphylococcus aureus. Infect. Immun. 1:169-173.

23. Deora, R., and T.K. Misra. 1996. Characterization of the primary sigma factor of Staphylococcus aureus. J. Biol. Chem. 271:2182821834.

24. Deora, R., T. Tseng, and T.K. Misra. 1997. Alternative transcription factor $\sigma^{\mathrm{SB}}$ of Staphylococcus aureus: characterization and role in transcription of the global regulatory locus sar. J. Bacteriol. 179: 6355-6359.

25. Derre, I., G. Rapoport, and T. Msadek. 1999. CtsR, a novel regulator of stress and heat shock response, controls clp and molecular chaperone gene expression in Gram-positive bacteria. Mol. Microbiol. 31:117-131.

26. Dubrac, S., P. Bisicchia, K.M. Devine, and T. Msadek. 2008. A matter of life and death: cell wall homeostasis and the WalKR (YycGF) essential signal transduction pathway. Mol. Microbiol. 70:1307-1322.

27. Foster, T.J. 2005. Immune evasion by staphylococci. Nat. Rev. Microbiol. 3:948-958.

28. Frees, D., A. Chastanet, S. Qazi, K. Sorensen, P. Hill, T. Msadek, and H. Ingmer. 2004. Clp ATPases are required for stress tolerance, intracellular replication and biofilm formation in Staphylococcus aureus. Mol. Microbiol. 54:1445-1462.

29. Fujimoto, D.F., R.H. Higginbotham, K.M. Sterba, S.J. Maleki, A.M. Segall, M.S. Smeltzer, and B.K. Hurlburt. 2009. Staphylococcus aureus SarA is a regulatory protein responsive to redox and $\mathrm{pH}$ that can support bacteriophage lambda integrase-mediated excision/ recombination. Mol. Microbiol. 74:1445-1458.

30. Gotoh, Y., A. Doi, E. Furuta, et al. 2010. Novel antibacterial compounds specifically targeting the essential WalR response regulator. J. Antibiot. (Tokyo) 63:127-134.

31. Gould, R.M., and W.J. Lennarz. 1970. Metabolism of phosphatidyl- 
glycerol and lysyl phosphatidylglycerol in Staphylococcus aureus. J. Bacteriol. 104:1135-1144.

32. Grant, R.A., D.J. Filman, S.E. Finkel, R. Kolter, and J.M. Hogle. 1998. The crystal structure of Dps, a ferritin homolog that binds and protects DNA. Nat. Struct. Biol. 5:294-303.

33. Gruber, T.M., and C.A. Gross. 2003. Multiple sigma subunits and the partitioning of bacterial transcription space. Annu. Rev. Microbiol. 57:441-466.

34. Hayami, M., A. Okabe, K. Sasai, H. Hayashi, and Y. Kanemasa. 1979. Presence and synthesis of cholesterol in stable staphylococcal L-forms. J. Bacteriol. 140:859-863.

35. Herbert, S., A. Bera, C. Nerz, D. Kraus, A. Peschel, C. Goerke, M. Meehl, A. Cheung, and F. Götz. 2007. Molecular basis of resistance to muramidase and cationic antimicrobial peptide activity of lysozyme in staphylococci. PLoS Pathog. 3:e102.

36. Hiramatsu, K., L. Cui, M. Kuroda, and T. Ito. 2001. The emergence and evolution of methicillin-resistant Staphylococcus aureus. Trends Microbiol. 9:486-493.

37. Imlay, J.A. 2003. Pathways of oxidative damage. Annu. Rev. Microbiol. 57:395-418.

38. Inose, Y., S.L. Takeshita, T. Hidaka, M. Higashide, A. Maruyama, H. Hayashi, K. Morikawa, and T. Ohta. 2006. Genetic characterization of the natural SigB variants found in clinical isolates of Staphylococcus aureus. J. Gen. Appl. Microbiol. 52:259-271.

39. Ito, T., K. Okuma, X.X. Ma, H. Yuzawa, and K. Hiramatsu. 2003. Insights on antibiotic resistance of Staphylococcus aureus from its whole genome: genomic island SCC. Drug Resist. Updat. 6:41-52.

40. Kiem, S., W.S. Oh, K.R. Peck, et al. 2004. Phase variation of biofilm formation in Staphylococcus aureus by IS256 insertion and its impact on the capacity adhering to polyurethane surface. J. Korean Med. Sci. 19:779-782.

41. Kim, J., S.H. Yoshimura, K. Hizume, R.L. Ohniwa, A. Ishihama, and K. Takeyasu. 2004. Fundamental structural units of the Escherichia coli nucleoid revealed by atomic force microscopy. Nucleic Acids Res. 32:1982-1992.

42. Kuroda, M., D. Kobayashi, K. Honda, H. Hayashi, and T. Ohta. 1999. The $h s p$ operons are repressed by the $h r c 37$ of the $h s p 70$ operon in Staphylococcus aureus. Microbiol. Immunol. 43:19-27.

43. Kuroda, M., H. Kuroda, T. Oshima, F. Takeuchi, H. Mori, and K. Hiramatsu. 2003. Two-component system VraSR positively modulates the regulation of cell-wall biosynthesis pathway in Staphylococcus aureus. Mol. Microbiol. 49:807-821.

44. Lightbown, J.W., and F.L. Jackson. 1956. Inhibition of cytochrome systems of heart muscle and certain bacteria by the antagonists of dihydrostreptomycin: 2-alkyl-4-hydroxyquinoline N-oxides. Biochem. J. 63:130-137.

45. Liu, C.I., G.Y. Liu, Y. Song, F. Yin, M.E. Hensler, W.Y. Jeng, V. Nizet, A.H. Wang, and E. Oldfield. 2008. A cholesterol biosynthesis inhibitor blocks Staphylococcus aureus virulence. Science 319:13911394.

46. Liu, G.Y., A. Essex, J.T. Buchanan, V. Datta, H.M. Hoffman, J.F. Bastian, J. Fierer, and V. Nizet. 2005. Staphylococcus aureus golden pigment impairs neutrophil killing and promotes virulence through its antioxidant activity. J. Exp. Med. 202:209-215.

47. López, C.S., A.F. Alice, H. Heras, E.A. Rivas, and C. Sánchez-Rivas. 2006. Role of anionic phospholipids in the adaptation of Bacillus subtilis to high salinity. Microbiology 152:605-616.

48. Maamar, H., A. Raj, and D. Dubnau. 2007. Noise in gene expression determines cell fate in Bacillus subtilis. Science 317:526-529.

49. Maresso, A.W., and O. Schneewind. 2006. Iron acquisition and transport in Staphylococcus aureus. Biometals 19:193-203.

50. Martinez, A., and R. Kolter. 1997. Protection of DNA during oxidative stress by the nonspecific DNA-binding protein Dps. J. Bacteriol. 179:5188-5194.

51. Minnikin, D.E., and H. Abdolrahimzadeh. 1974. Effect of pH on the proportions of polar lipids, in chemostat cultures of Bacillus subtilis. J. Bacteriol. 120:999-1003.

52. Mitchell, G., E. Brouillette, D.L. Séguin, A.-E. Asselin, C.L. Jacob, and F. Malouin. 2010. A role for sigma factor B in the emergence of Staphylococcus aureus small-colony variants and elevated biofilm production resulting from an exposure to aminoglycosides. Microb. Pathog. 48:18-27.

53. Mitchell, G., D.L. Séguin, A.-E. Asselin, E. Déziel, A.M. Cantin, E.H. Frost, S. Michaud, and F. Malouin. 2010. Staphylococcus aureus sigma B-dependent emergence of small-colony variants and biofilm production following exposure to Pseudomonas aeruginosa 4hydroxy-2-heptylquinoline- $N$-oxide. BMC Microbiol. 10:33.

54. Moeller, R., P. Setlow, G. Reitz, and W.L. Nicholson. 2009. Roles of small, acid-soluble spore proteins and core water content in survival of Bacillus subtilis spores exposed to environmental solar UV radiation. Appl. Environ. Microbiol. 75:5202-5208.

55. Mooney, R.A., S.A. Darst, and R. Landick. 2005. Sigma and RNA polymerase: an on-again, off-again relationship? Mol. Cell 20:335345 .

56. Morikawa, K., Y. Inose, H. Okamura, A. Maruyama, H. Hayashi, K. Takeyasu, and T. Ohta. 2003. A new staphylococcal sigma factor in the conserved gene cassette: functional significance and implication for the evolutionary processes. Genes Cells 8:699-712.

57. Morikawa, K., R.L. Ohniwa, J. Kim, A. Maruyama, T. Ohta, and K. Takeyasu. 2006. Bacterial nucleoid dynamics: oxidative stress response in Staphylococcus aureus. Genes Cells 11:409-423.

58. Morikawa, K., R.L. Ohniwa, J. Kim, S.L. Takeshita, A. Maruyama, Y. Inose, K. Takeyasu, and T. Ohta. 2007. Biochemical, molecular genetic, and structural analyses of the staphylococcal nucleoid. Microsc. Microanal. 13:30-35.

59. Msadek, T. 1999. When the going gets tough: survival strategies and environmental signaling networks in Bacillus subtilis. Trends Microbiol. 7:201-207.

60. Nagamachi, E., Y. Hirai, K. Tomochika, and Y. Kanemasa. 1992. Studies on osmotic stability of liposomes prepared with bacterial membrane lipids by carboxyfluorescein release. Microbiol. Immunol. $36: 231-234$

61. Nagy, Z., and M. Chandler. 2004. Regulation of transposition in bacteria. Res. Microbiol. 155:387-398.

62. Nair, S., and S.E. Finkel. 2004. Dps protects cells against multiple stresses during stationary phase. J. Bacteriol. 186:4192-4198.

63. Nishiyama, Y., and H. Yamaguchi. 1990. Morphological detection of filipin-sterol complexes in the cytoplasmic membrane of staphylococcal L-form. Microbiol. Immunol. 34:25-34.

64. Novick, R.P. 2003. Mobile genetic elements and bacterial toxinoses: the superantigen-encoding pathogenicity islands of Staphylococcus aureus. Plasmid 49:93-105.

65. Ohniwa, R.L., K. Morikawa, J. Kim, T. Ohta, A. Ishihama, C. Wada, and K. Takeyasu. 2006. Dynamic state of DNA topology is essential for genome condensation in bacteria. Embo J. 25:5591-5602.

66. Ohniwa, R.L., K. Morikawa, S.L. Takeshita, J. Kim, T. Ohta, C. Wada, and K. Takeyasu. 2007. Transcription-coupled nucleoid architecture in bacteria. Genes Cells 12:1141-1152.

67. Ohniwa, R.L., K. Morikawa, C. Wada, T. Ohta, and K. Takeyasu. 2010. Nucleoid architecture and dynamics in bacteria. In W.D. Knudsen and S.S. Bruns (ed.), Bacterial DNA, DNA polymerase and DNA helicases. Nova Science Publishers, Hauppauge.

68. Ohta, T., S. Nettikadan, F. Tokumasu, H. Ideno, Y. Abe, M. Kuroda, H. Hayashi, and K. Takeyasu. 1996. Atomic force microscopy proposes a novel model for stem-loop structure that binds a heat shock protein in the Staphylococcus aureus HSP70 operon. Biochem. Biophys. Res. Commun. 226:730-734.

69. Otto, M. 2008. Staphylococcal biofilms. Curr. Top. Microbiol. Immunol. 322:207-228.

70. Peschel, A. 2002. How do bacteria resist human antimicrobial peptides? Trends Microbiol. 10:179-186.

71. Proctor, R.A., C. von Eiff, B.C. Kahl, K. Becker, P. McNamara, M. Herrmann, and G. Peters. 2006. Small colony variants: a pathogenic form of bacteria that facilitates persistent and recurrent infections. Nat. Rev. Microbiol. 4:295-305.

72. Ragolia, L., and B.E. Tropp. 1994. The effects of phosphoglycerides on Escherichia coli cardiolipin synthase. Biochim. Biophys. Acta 1214:323-332.

73. Ramos, J.L., E. Duque, M.T. Gallegos, P. Godoy, M.I. RamosGonzalez, A. Rojas, W. Teran, and A. Segura. 2002. Mechanisms of solvent tolerance in gram-negative bacteria. Annu. Rev. Microbiol. 56:743-768.

74. Romantsov, T., Z. Guan, and J.M. Wood. 2009. Cardiolipin and the osmotic stress responses of bacteria. Biochim. Biophys. Acta. 1788:2092-2100.

75. Rosdahl, V.T., and R. Vejlsgaard. 1970. Investigation of the penicillinase activity in L colonies of Staphylococcus aureus. Appl. Microbiol. 20:871-874. 
76. Sato, H., and T. Ohya. 1987. Studies on biological characteristics of staphylococcal L-forms. Bulletin of the Faculty of Agriculture, Kagoshima University 37:167-174.

77. Schlame, M. 2008. Cardiolipin synthesis for the assembly of bacterial and mitochondrial membranes. J. Lipid. Res. 49:1607-1620.

78. Setlow, P. 2006. Spores of Bacillus subtilis: their resistance to and killing by radiation, heat and chemicals. J. Appl. Microbiol. 101:514525 .

79. Shaw, L.N., C. Lindholm, T.K. Prajsnar, H.K. Miller, M.C. Brown, E. Golonka, G.C. Stewart, A. Tarkowski, and J. Potempa. 2008. Identification and characterization of sigma, a novel component of the Staphylococcus aureus stress and virulence responses. PLoS ONE 3:e3844.

80. Short, S.A., and D.C. White. 1971. Metabolism of phosphatidylglycerol, lysylphosphatidylglycerol, and cardiolipin of Staphylococcus aureus. J. Bacteriol. 108:219-226.

81. Smith, J.A., and A.T. Willis. 1967. Some physiological characters of L forms of Staphylococcus aureus. J. Pathol. Bacteriol. 94:359-365.

82. Süel, G.M., J. Garcia-Ojalvo, L.M. Liberman, and M.B. Elowitz. 2006. An excitable gene regulatory circuit induces transient cellular differentiation. Nature 440:545-550.

83. Taber, H.W., J.P. Mueller, P.F. Miller, and A.S. Arrow. 1987. Bacterial uptake of aminoglycoside antibiotics. Microbiol. Rev. 51:439-457.

84. Takeyasu, K., J. Kim, R.L. Ohniwa, T. Kobori, Y. Inose, K. Morikawa, T. Ohta, A. Ishihama, and S.H. Yoshimura. 2004. Genome architecture studied by nanoscale imaging: analyses among bacterial phyla and their implication to eukaryotic genome folding. Cytogenet. Genome Res. 107:38-48.

85. Valle, J., M. Vergara-Irigaray, N. Merino, J.R. Penades, and I. Lasa. 2007. sigmaB regulates IS256-mediated Staphylococcus aureus biofilm phenotypic variation. J. Bacteriol. 189:2886-2896.

86. van Schaik, W., and T. Abee. 2005. The role of sigmaB in the stress response of Gram-positive bacteria - targets for food preservation and safety. Curr. Opin. Biotechnol. 16:218-224.

87. Wieland, B., C. Feil, E. Gloria-Maercker, G. Thumm, M. Lechner, J.M. Bravo, K. Poralla, and F. Gotz. 1994. Genetic and biochemical analyses of the biosynthesis of the yellow carotenoid 4,4'-diaponeurosporene of Staphylococcus aureus. J. Bacteriol. 176:7719-7726.

88. Wisniewski-Dyé, F., and L. Vial. 2008. Phase and antigenic variation mediated by genome modifications. Antonie Van Leeuwenhoek 94:493-515.

89. Wolf, S.G., D. Frenkiel, T. Arad, S.E. Finkel, R. Kolter, and A. Minsky. 1999. DNA protection by stress-induced biocrystallization. Nature 400:83-85.

90. Wösten, M.M.S.M. 1998. Eubacterial sigma-factors. FEMS Microbiol. Rev. 22:127-150.

91. Yamamoto, Y., L.B. Poole, R.R. Hantgan, and Y. Kamio. 2002. An iron-binding protein, Dpr, from Streptococcus mutans prevents iron-dependent hydroxyl radical formation in vitro. J. Bacteriol. 184:2931-2939.

92. Yarwood, J.M., K.M. Paquette, I.B. Tikh, E.M. Volper, and E.P. Greenberg. 2007. Generation of virulence factor variants in Staphylococcus aureus biofilms. J. Bacteriol. 189:7961-7967. 